\title{
STUDY ON URBAN RAIL RAPID TRANSIT ACCIDENTS PREVENTION AND HAZARDS IDENTIFICATION
}

\author{
Lung-Chuang Wang \\ Department of Civil Engineering, National Taipei University of Technology, \\ Taipei, Taiwan, R.O.C.
}

\begin{abstract}
In comparison with the other transportation modes, rail rapid transit already enjoys a higher degree of safety as it is designed with the exclusive right of way and the automatic train control and monitoring systems. Nevertheless, rail rapid transit systems are not free from serious accidents, which often led to human injuries and facility damages. The planning of accident prevention and emergency measures, therefore, are still important issues of the rail rapid transit operations. In this study, we examine the hazard identification model in the rail rapid transit by reviewing the theoretical basis on hazards and accidents, and analyzing on the historical rail rapid transit accident data and the rail rapid transit safety features. Fourteen categories of rail rapid transit accidents are classified and listed by their nature and cause. The model presented in this study analyses the process of hazard identification in two stages, which clearly indicate the factors of the inherent and direct hazard and the weaknesses in the response system. The study uses the fire accident took place in the Mucha Line of Taipei Rapid Transit System in 1993 as an example to illustrate the hazard identification model by pointing out the cause of the incident and the weakness in the emergency systems.
\end{abstract}

Keywords: Rail Rapid transit, Accidents analysis, Hazard identification, Accident causation, Case study

\section{INTRODUCTION}

Rail rapid transits have already become an indispensable means of public transportation for modern cities. To date, there are hundreds of rail rapid transit systems located in different parts of the world. While these systems have provided solutions for a fast, comfortable, reliable, and convenient means of transportation for travels in the urban areas, the rail rapid transit is also expected to provide comprehensive measures to ensure the operation safety. In comparison with the other transportation modes, rail rapid transit already enjoys a higher degree of safety as it is designed with the exclusive right of way and the automatic train control and monitoring systems. Still, rail rapid transit systems are not free from serious accidents, which often lead to human injuries and facility damages and may result in secondary disasters (e.g., heavy injuries may be incurred by electrical shocks in the confusion of evacuation). According to the statistics from FTA [1], the heavy rail transit suffered a fatality rate of 0.78 per hundred million passenger-miles for patrons and non-patrons fatalities. To prevent such casualties from occurring often involve some difficult issues, e.g., the control of operators and passengers and the constraints of the facility and the local environment. Perhaps, it is inevitable that "mishandling are prone to occur whenever manual operation is involved; failures are inevitable whenever machines are employed." The planning of accident prevention and emergency measures, therefore, are important issues of the rail rapid transit operation.

The chief objective of this study is to identify the hazards of the rail rapid transit accidents to prevent disaster from occurring. The rail rapid transit mentioned in this study includes the heavy and light rail rapid transit, which has the exclusive right of way, grade separation and the automatic train control and monitoring systems. The "accident" could be termed as unpredictable and unexpected, but in this study, the so-called "accidents" have the causes readily identifiable; only the precise moment when their effects will be noticed is unknown. Therefore, "accident" is not the target; instead, the hazards that cause the unwanted harmful event are the major concern in this study. 


\section{THEORETICAL REVIEWS}

The definition of hazard is "the source of energy and the physiological and behavioral factors which, when uncontrolled, lead to harmful occurrences." [2] whereas the definition of accident is "any occurrence that interrupts or interferes with the orderly progress of the activity in question.'[3] In other words, accidents do not necessarily incur human injuries; some accidents that cause damages and the others that do not inflict any losses. The scope of this study is targeted on the accidents that have resulted in human injuries and equipment damages. The following is a theoretical review on the causes of accidents and the safety features of rail rapid transit.

\subsection{Causes of Accidents}

In general, accidents happen because the place of the activity or the nature of the activity is dangerous. Moreover, they are induced to happen by external elements. However, before they take place, they have mostly become abnormal state, and eventually turn out to be accidents. Without proper control and handling, accidents will result in injuries or disasters.

Florio, Alles and Stafford [4] have stated that all hazardous accidents are environmental hazards and unsafe behavior. Grimaldi and Simonds [2] divided the causes of injurious accidents into two categories: unsafe physical conditions and unsafe personal acts. The classifications for each category are suggested to refer to their book.

In the early edition of Heinrich's book [5], he had stated that the main causes of hazardous accidents are unsafe behavior of men, or mechanical and physical unsafe conditions. Secondary causes are due to defects of personnel or social environmental elements. His statement further explains chain reactions (domino reaction) of these elements. With proper control of the key domino of unsafe behavior and unsafe conditions, accident and injury will not happen. .

\subsection{Safety Features}

Safety features on the rail rapid transit can be examined from three aspects, including system, operating staff, and environment.

System. Rail rapid transit's system facility is numerous and complicated and requires high degree of professional training for operation.

(1) Extensive operation control and monitoring. Operation is carefully monitored by ATC (Automatic Train Control), ATO (Automatic Train Operation), ATP (Automatic Train Protection), and ATS (Automatic Train
Supervision)

(2) Fail-safe design principle. All the safety related facilities are required to design according to the fail-safe principle, which means the system could still operate safely in case of system failure.

(3) The power supply system is operating on a continuous and stable basis.

Operating Staff. All the staff must be given proper training and obtain certification to be admitted. The medical condition and technical expertise of every operating staff are examined routinely. The safety organization of the transit authority handles the planning, assessing, and implementing of the safety program, which well defined the responsibility and treatment of emergent happenings.

Environment. Rail rapid transit has the exclusive right of way which either through underground tunnels or overhead viaducts. This exclusive right of way prohibits the train from external impact so that the rail rapid transit will be much safer than other transportation mode. Nevertheless, heavy losses can be anticipated when accidents occur in these sections. That is why it needs extensive operation control and monitoring systems and well trained operating staff.

\section{CATEGORIES OF RAIL RAPID TRANSIT ACCIDENTS}

This paper has collected 46 case studies from the United States and Taiwan (NTSB [6], BART [7], Wang [8] ), which can be divided into 14 different categories by their nature and the cause of accidents.

\subsection{Fire}

Fire has the highest rate of occurrence among all rail rapid transit hazards and it often results in the most damaging disaster. In addition, fire is frequently identified as the cause of secondary damages. The most frequent causes of fire are as follows.

(1) Arson. Arson includes deliberate and unintentional act of setting a fire.

(2) Failure of Electrical Equipment. Failure of the electrical equipment often leads to fire as seen in: (a) Escalators may cause fire due to overloading or lack of maintenance. (b) Heating, air conditioning, and ventilation systems due to overheating and poor power distribution design. (c) Lamps, switches, and fuse could also cause fire if they are shortcircuited. (d) Motor Control Group is identified as the most frequent cause of fire in the rolling stock. When the protective cover is 
not covered properly, it could scrape against the third rail power supply equipment or other electrical parts and ignite fire. Negligence in maintaining the switchboard, metal duct, and cable could also result in fire during operation. (e) Current collector's components could be installed properly causing it to scrape against the bogie's frame and resulting in fire. (f) Overheat of resistor grid; (g) Short circuit of battery cable. (h) Metallic objects lodged under car may not be secure in place causing it to rub against other electrical equipment and result in fire. (i) Insulation material on the power supply cable may be rubbed off, which leaves the cable much more susceptible to become a medium of fire. (j) Sparks in the third rail may light up the inflammable material on the track and cause fire.

(3) Brakes. Either mishandling or equipment failure could lead to fire.

(4) Storage of inflammable material is improper.

(5) When renovation is in progress, fire could be caused by disposal of cigarette butts by the workers or by the presence of inflammable material such as paint, chemicals, or sawdust.

(6) Derailing can often lead to fire.

(7) Fire can also be caused by the strike of lightening on the electrical equipment. Such occurrence is more frequently seen in the tropical areas.

\subsection{Flood}

Such hazard is seldom reported in rail rapid transit accidents; the only exception is found in the underground rail rapid transit. The failure of the drainage system could easily lead to flood. Once the flood reaches the third rail power supply system or any of the on-board electrical equipment, it could result in short circuiting.

\subsection{Collisions}

Possible causes for such accidents are (1) Derailed train-crashing head on with the approaching train traveling on the other track. (2) Violation of stop signs by the driver (intrusion). (3) Violation of speed by the driver (speeding). (4) Failure of the ATC system. (5) Signal errors, e.g., lost of signal or computer operation errors. (6) Breakdown of the split switch. (7) Mishandling of the split switch.

\subsection{Derailment}

Factors contributing to derailment are as follows: (1) Mishandling or failure of the split switch. (2) Incomplete release of hand brake which makes the wheels to overheat and deform and eventually result in derailment; (3) Inadequate design of the geometric design (e.g., inadequate curvature or uneven elevation of the track); (4) Excessive rocking of the trains (due to the uneven ground or tracks) that causes the vehicle to scrape against the train traveling in the opposite direction and eventually resulting in derailment. (5) Speeding at the turn. (6) Excessive wearing on the steel wheel. (7) Uneven track (e.g., sinking of the foundation, especially prone to occur in the area of soft foundation). (8) Damage of the bearing and wheel. (9) Rail deformation (e.g., caused by earthquake or loosening of rail.)

\subsection{Door Accident}

Such accidents normally only involve a single passenger and it is especially prone to take place during peak hours with large number of commuting crowd on board. The result of crowding can often lead to jamming at the door, or failure for the door to open or to close.

\subsection{Breakdowns of power supply system}

Breakdown of the power supply system will cause the train in motion to stop abruptly. The causes of power failure include failure of the power supply or emergency power supply facilities, overload, mishandling, and struck by lightening.

\subsection{Intrusion}

Either animals or people could be found intruding the track. Most of these incidences took place on the level ground are apt to cause considerable damages during the peak hours where there are a large number of trains on the track.

\subsection{Electric Shocks}

These accidents usually take place on the open ground or at the rail yard. When the insulation material on the third rail become detached, the maintenance staff may be struck by electric shocks while maintaining the vehicle.

\subsection{Crowding}

Excessive loading of passengers during peak hours may cause passenger to be jammed or have heart failure or shocks.

\subsection{Gap fall}

The likely locations for this type of incidence to take place include stairway, the edge of the platform, and escalators. According to the San Francisco rail rapid transit statistics, there are 157 counts of gap falls accidents taking place between 1985 and 1987 [7].

\subsection{Scraped by the train}

Either because the passenger is mindless of the dangers or there is a lack of proper signs on the platform, accidents may take place as the train pulls in the station and scrapes the passenger that stands too close to the edge of the platform. Depending on the force of contact, the passenger may be pulled off 
the platform unto the track and cause more serious injuries.

\subsection{Suicide}

With the advent of the industrialized society, the number of people committing suicide is on the increase, which are both difficult to prevent and predict. According to the San Francisco rapid transit statistics, there are 13 suicidal accidents from 1985 to 1987 [7]. In 1995, suicides were reported separately in the FTA's database and accounted for one-third of rail transit fatalities [1].

\subsection{Criminal Acts}

Deliberate actions to destroy the system facility or set up are the major concern in this respect. On the other hand, some criminal actions happened at the train or station also caused the passengers to get serious injuries. Although such kinds of security problems are difficult to prevent, they do have important relation with the safety management of the rail rapid transit systems.

\subsection{Natural Disaster}

Accidents caused by forces in nature such as earthquakes, lightening, storms, flood, and heat waves are described in the following.

(1) Earthquake. Severe earthquake can cause structural damages to the rail rapid transit system, e.g., collapse of the structure and damage on the system. Even less severe earthquake can cause hidden damage to the structure or to the system. This may become the leading cause of hazards in later days.

(2) Lightening. The power supply system on the viaduct is especially susceptible to lightening strike.

(3) Storm. Ground levels and the viaduct sections of the rail rapid transit line are more likely to suffer from the storm.

(4) Heat Waves. Excessive heat will not only increase the mishandling rate in manual operation; it also causes deformation of the track, which may lead to serious accidents such as derailment and fires.

\section{RAIL RAPID TRANSIT HAZARD IDENTIFICATION MODEL}

Given the information presented previously, the rail rapid transit hazard identification model is introduced as follows. This model first establishes the cause-effect charts of hazardous factors that lead to unsafe conditions and then a two-stage analysis is adapted to identify the hazards of the respective incidents or accidents.
Generally, rail rapid transit hazards occur because of the three elements: (1) Human: operators and passengers; (2) System: material, equipment, tools, and safety facility; (3) Environment: temperature, humidity, ventilation, lights, and noises (natural environment and artificial environment) in the working place.

If we analyze specifically about the causes that lead to system and environmental unsafe conditions, we find that almost all defects come from human errors in design, installation, operation, treatment, and uses. Even the defects in natural environment can be reduced or prevented by pre-alarm system and preventive measures. Though the human error could be regarded as the major cause of rail rapid transit accident, the human behaviors also result from the transit equipment, facilities, and the other environmental factors.

\subsection{Establishing the Cause-Effect Relationship}

Contributing factors for the cause of rail rapid transit accidents can be classified as human induced, system induced, or environmentally induced. Most of the accidents, however, are not caused by a single factor, there are usually a cluster of factors interacted with each other. As the three groups of factors bear influences on one another, it is important to devise a scheme that can manage the consequence of the three elements and recognize hazardous conditions to ensure the operation safety. The following Fig. 1 to Fig. 3 are the fishbone charts that this study uses to provide cause-effect relationship of how these factors lead to hazards. In these figures, the large-boned branches are the indirect causes and the sub-branches are the direct causes, which both contribute to one of these main factors and will lead to unsafe behaviors or unsafe conditions.

\subsection{Human Induced Factors.}

Human induced accidents could be attributed to either the operators or the passengers, but the operators are more likely to be the media of accidents. Factors leading to operator induced accidents include (a) Physical and mental conditions. Fatigue or other illness may cause the operators to perform erroneous procedure. Unstable mental state could also influence normal operation; (b) Immature operating skills; (c) Failure to follow the operation procedure; (d) Lack of safety knowledge and awareness. Factors leading to the passenger-induced accidents are (e) Illegal actions; (f) Mindless of the dangers; (g) Deliberate act; (h) Deficient in common sense. The above factors are indicated at the largeboned branches of Fig. 1.

\subsection{System induced Factors}

\subsection{Elements of Hazards Identification}


The rail rapid transit system is composed of equipment (rolling stock, signaling, and power supply) and station facilities (platform, track, and others). The factors leading to the system induced accidents include (a) Inadequate selection of equipment. Examples include: capacity of the equipment could not satisfy the functional demand, the equipment is below the safety standard; and equipment cannot adapt to the unique environmental set up. (b) Inadequate installation of equipment; (c) Inadequate maintenance of equipment; (d) Equipment exceeding the design life cycle. (e) Inadequate application of equipment; (f) Inadequate design of equipment. These factors are indicated at the largeboned branches of Fig. 2.

\subsection{Environmentally Induced Factors}

This aspect can be further divided into the natural environment and the working environment.

Natural environment. Earthquakes, lightening, flood, typhoons, or heat waves may affect the transit operation.

Working environment. Working environment can be further divided into two parts, the software, and the hardware. Software includes organizational structure, management efficiency, remuneration, and labor relations. Hardware refers to the lighting, the ventilation, temperature, and humidity of the place at work. An efficient and integral operating organization is not only to the organization itself, but it also has direct influence on the control and prevention of the human-induced and system-induced hazards.

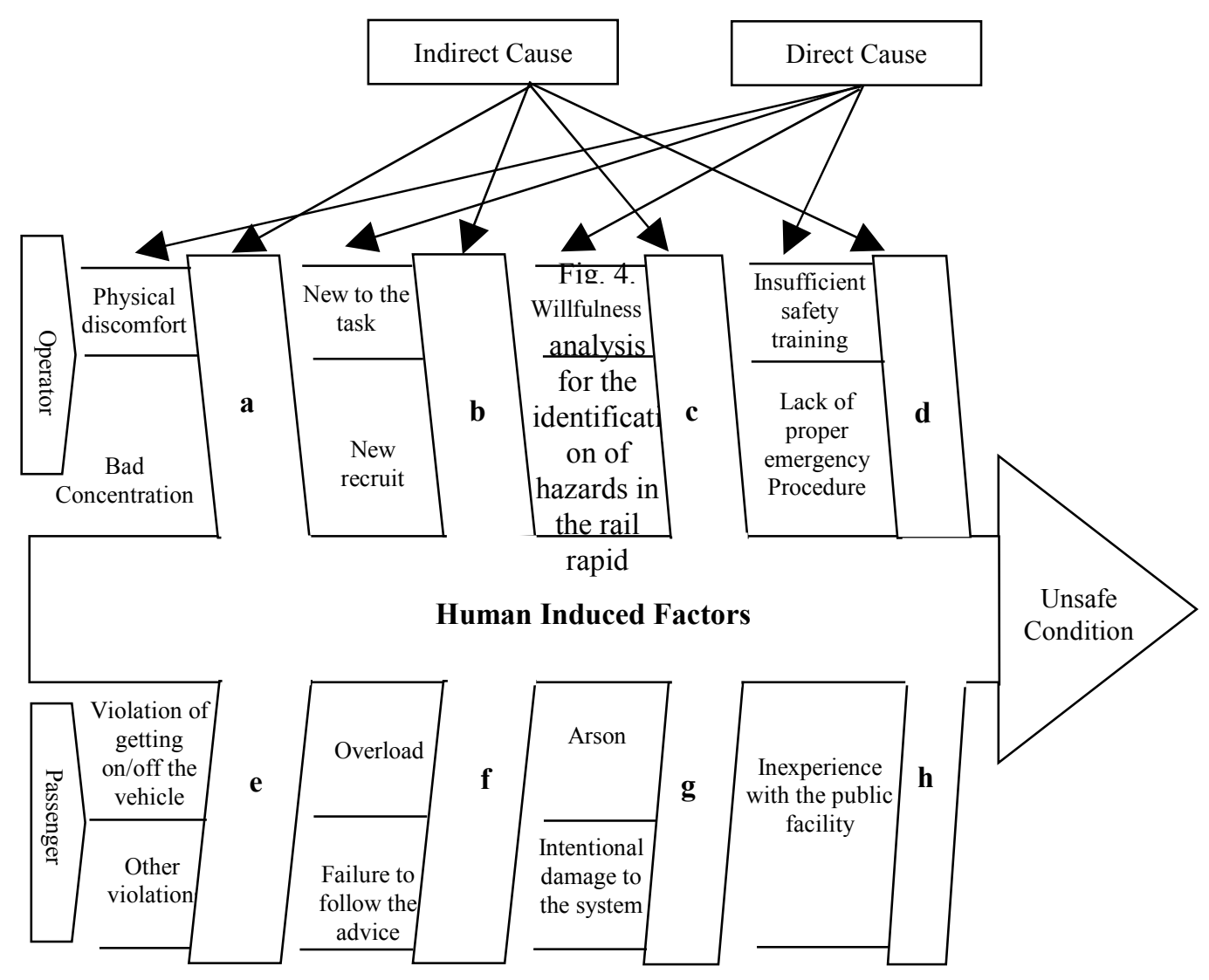

Fig. 1. Human induced hazards in rail rapid transit systems 


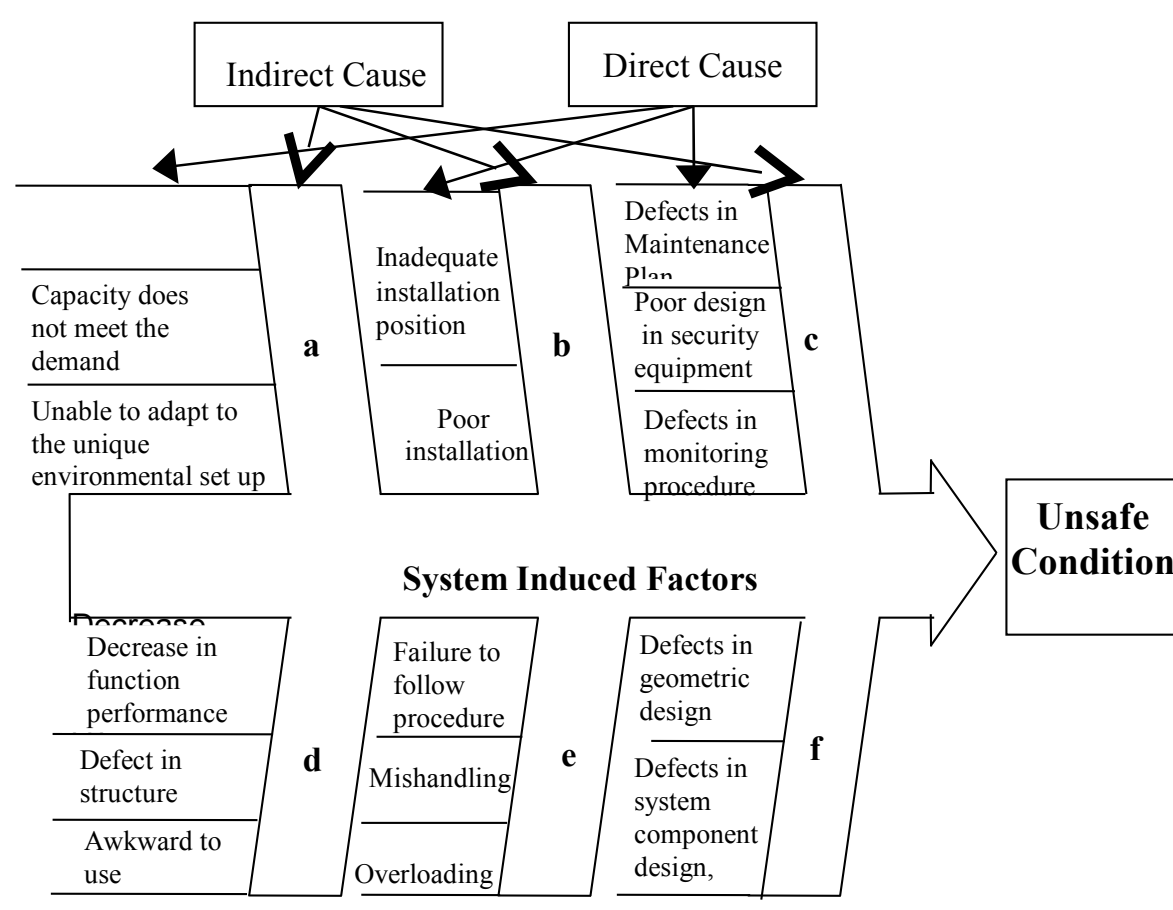

Fig. 2. System induced hazards in rail rapid transit systems

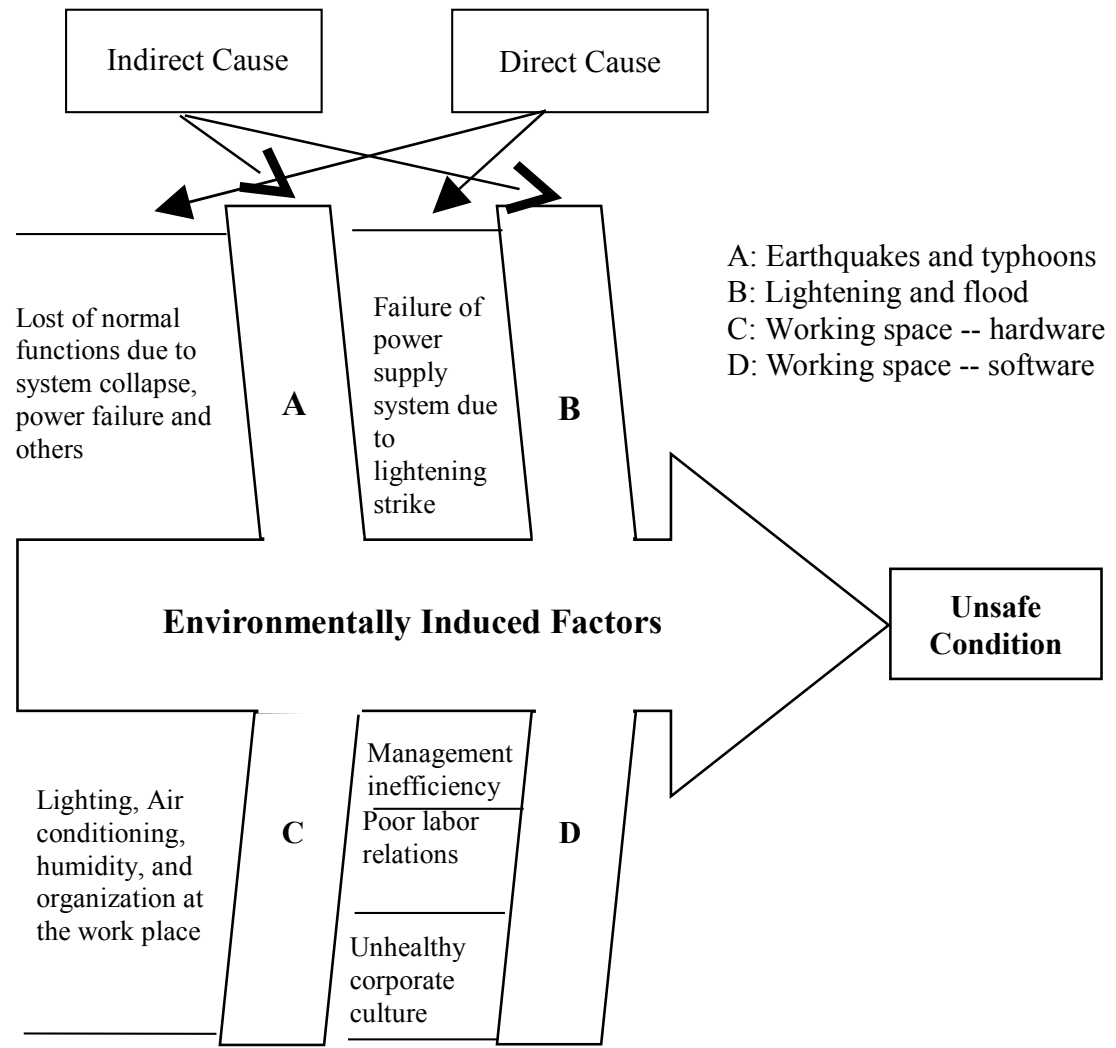

Fig. 3. Environmentally induced hazards in rail rapid transit systems 


\subsection{Two-Stage Analysis}

The process of rail rapid transit hazard identification can be described in two stages. This is depicted as in Fig. 4

The First Stage. Three potential hazardous (human-induced, system induced, and environmentally induced) factors would cause the system unsafe conditions, but they might not affect the normal operation immediately. In this case, most of the hazards can be eliminated under a perfect management system, for example: an accurate routine check system, the alertness of the operators, a good auditing system, and a strict quality and safety control system. With the application of the fishbone chart of human, system and environment induced factors, the specific unsafe conditions or behaviors can identify the inherent hazards.

The Second Stage. A medium that causes the potential hazard to become a contributing factor for the accident is also identified as a direct hazard. As for the rail rapid transit, all the safety design is targeted in preventing the formation of such media. Whereas the potential hazards are not eliminated in the first stage, the automatic train operation control and monitoring system will come into play. The Automatic Train Supervision (ATS) and Automatic Train Protection (ATP) should detect any abnormalities in the system and send the messages to the control center for correction, or to the driver to pull on the emergency brake. If the system involves no manual operation, i.e., a "driverless" system, the rate of removing errors through the automatic train control and monitoring system should be $100 \%$. Once the unsafe conditions keep on exposing, the hazards in the second stage will be identified by the factors that the automatic train control and monitoring system fail to respond with safety measures and stop the exposure of hazardous elements.

The two-stage analysis provides the first step to detect the causes of rail rapid transit accidents and composes the major part of the hazard identification model. To explain how to use it, we will demonstrate the process by the real case in next section.

\section{CASE STUDY}

Mucha line is the first completed and the only automatic guided rail rapid transit line in Taipei. Along the 10.9-kilometer route, there are 12 stations and a Mucha maintenance depot. The construction date started in 1988. Forecasted substantial completion date was originally August 2, 1993, which deferred to June 1994 for two serious fire accidents. Finally, the commercial operation starts at March 1996. Five serious accidents, which suspending train operation over one hour, were recorded for the first year of commissioning, which is way over the acceptable standard ( 0 accident per million $\mathrm{km}$ ). This study will take one of the two fire incidents, which took lace in September 24, 1993, as the case study to illustrate the causes of the accidents using the hazard identification model developed in this study.

Mucha line uses the Matra VAL 256 system, which is originally designed as a "driverless", twocoach (one married-pair) system, with one coach equipped with ATC system. The Department of Rapid Transit Systems of Taipei city government, however, demand the system to change to a two married-pair system to provide better service capacity

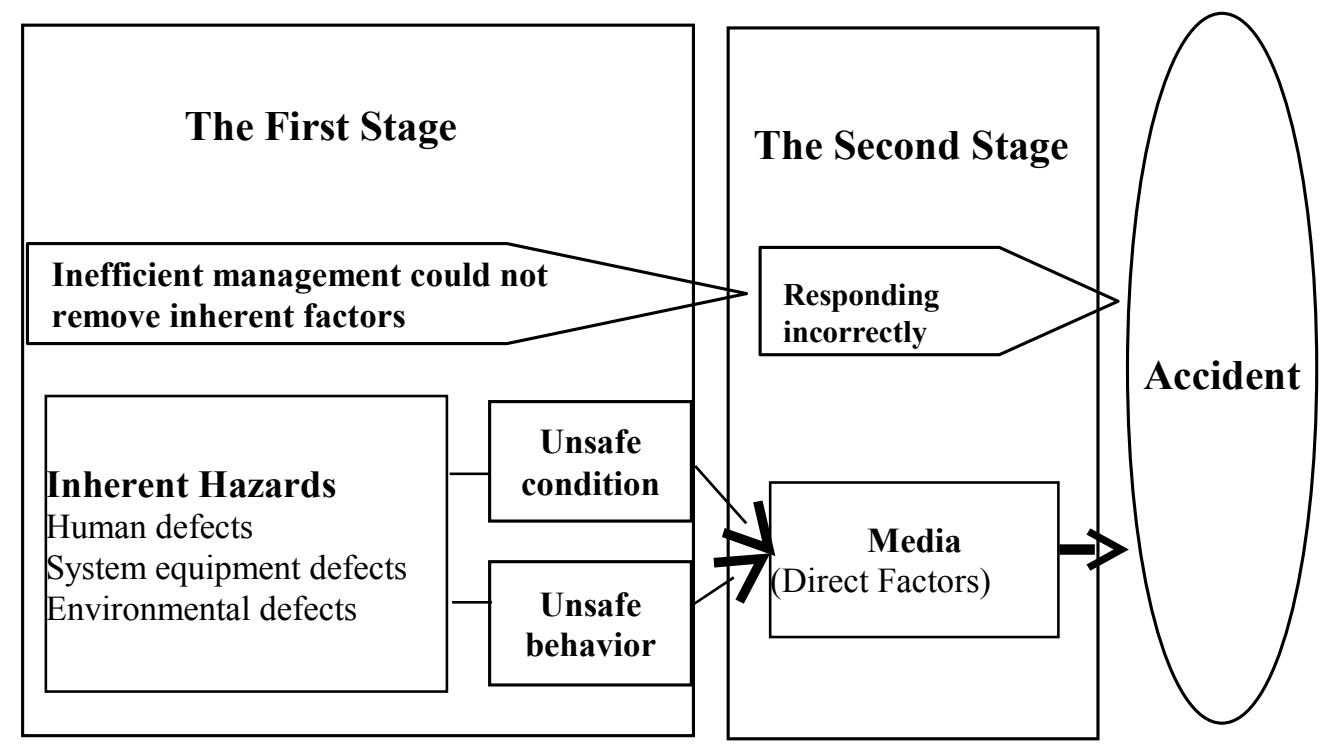

Fig. 4. Two-stage analysis for the identification of hazards in the rail rapid transit 
and operation flexibility with the condition that the new design should include operation safety guarantee, facility integration, and protection of passenger emergency evacuation. The VAL automatic control system in each married-pair is designed with the fail-safe principle and consisted of On-Board Automatic Train Control (OBATC), Fixed Automatic Train Control (Fixed ATC), Dwell Operation Control Unit (DOCU), Operation Control Center (OCC), and Safety Frequency \& High Voltage Logic [9].

After four month of the first fire incident, the second fire incident happened at about five o'clock in the morning of September 24 while the two marriedpair is driving back to maintenance depot under automatic train operation. The passer-by heard the explosion and saw the smoky train. The OCC did not aware of the situation until the witness informed the fire department. Though the OCC broke the circuit immediately, the train was burned in great fire that is higher than the roof of the train and clearly watched by the citizenry through the morning TV news. This event became the headlines and shocked the audience.

According to the press release of the accident report submitted by Matra for this event (quoted from the news reported by China Times, October 14, 1993): "The accident was induced by the failure of Traction Safety Rank (TSR) in the third coach of the two married-couple. Because the brake system was malfunctioned, it could not transmit the correct messages to the brake control system, which meant the automatic control system could not inform the other coaches to brake. A coach with the jammed brake was dragged forward by the other three coaches in motion was the cause of the accident." The failures of the Traction Safety Rank and the brake system are unsafe conditions that are result from several factors of hazard. Through the application of hazard identification model presented in last section, the consequence and factors of hazard within this accident are analyzed as follows and depicted as in Fig. 5.

(1) The First Stage. The unsafe conditions, such as the breakdown of the Traction Safety Rank and the brake system, are classified as system failure - an inherent cause of accident. These hazards induced by system factors could be identified as "Inadequate design and maintenance of equipment" and "Inadequate selection of equipment", which are indicated as the rightest branch in Fig.6. Another unsafe condition that the OCC fail to be aware of the incident immediately is the total effect of the three elements. The operation staff explained they received too many false messages at that time, so they turn off the alarm bell. This could be regarded as the "lack of safety knowledge and awareness". There might be insufficient safety training for the staff and the emergency procedure is defective. Furthermore, the corporate culture in the operation authority is not aware enough of the importance of safety.

(2) The Second Stage. After the brake was jammed, the train still ran for an hour and a half (about $2 \mathrm{~km}$ ) before the wheel was caught in fire. The medium for the fire accident was created in this stage, and the factors of hazard contributing to the creation of the medium are described as the following and indicated in Fig. 7.

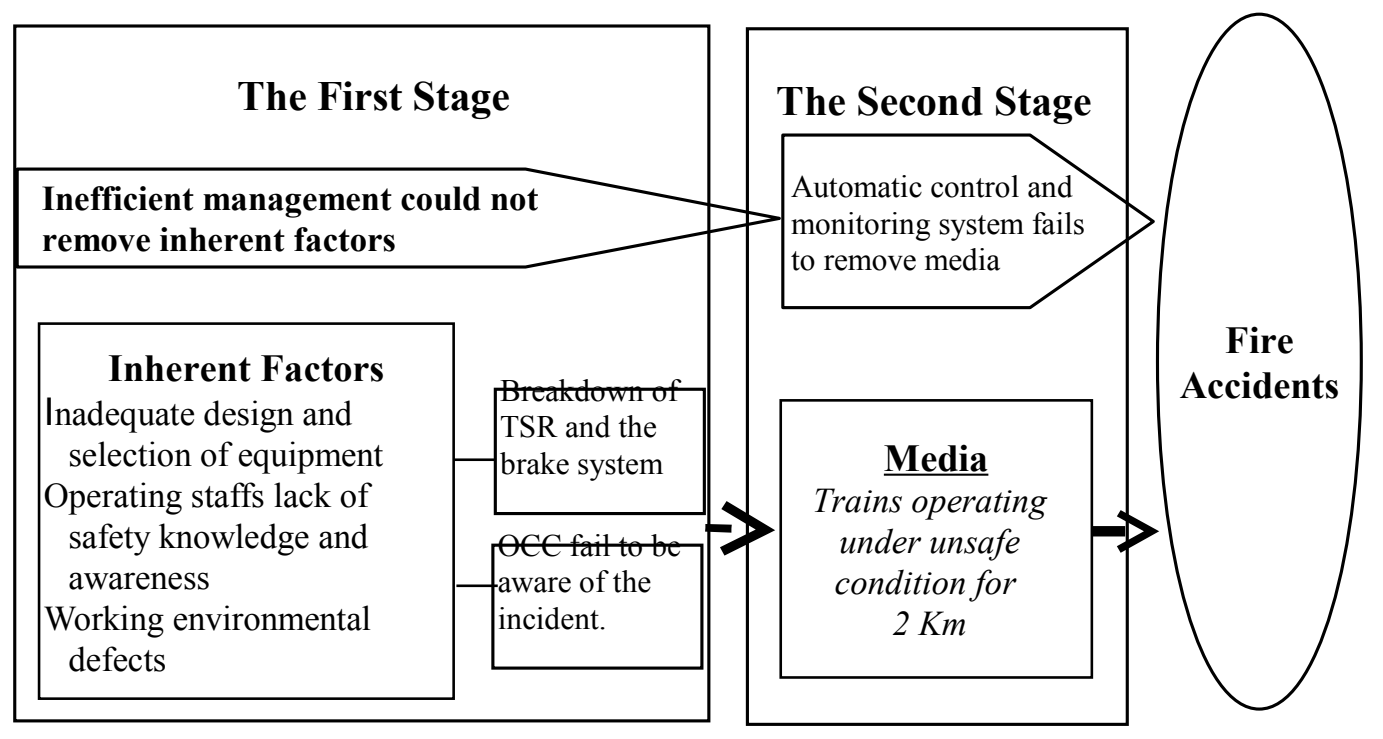

Fig. 5. Two-stage analysis for hazard identification in Mucha line 


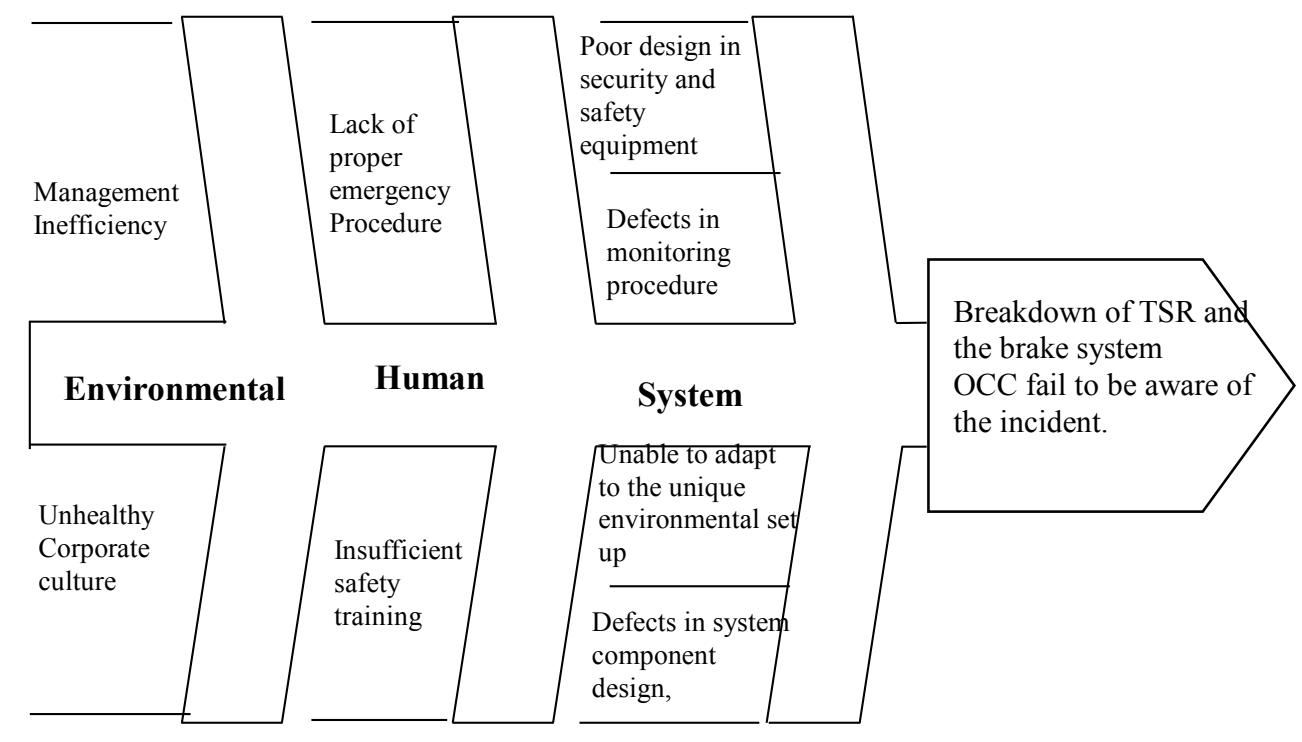

Fig. 6. Hazards identified in the first stage analysis

ATP did not detect any brake failure. No message was sent to the control center and no emergency measure was taken to correct the situation, hence, the creation of hazardous medium. There are three possible factors accounting for the ATP's failure to detect abnormalities: (a) There was a design defect in ATP; (b) There was a material or manufacturing defect, or (c) ATP was unable to function due to high temperature, magnetic or electric wave interference.

ATP had detected brake failure. Message was transmitted to the control center, but the response of the operator at the control center

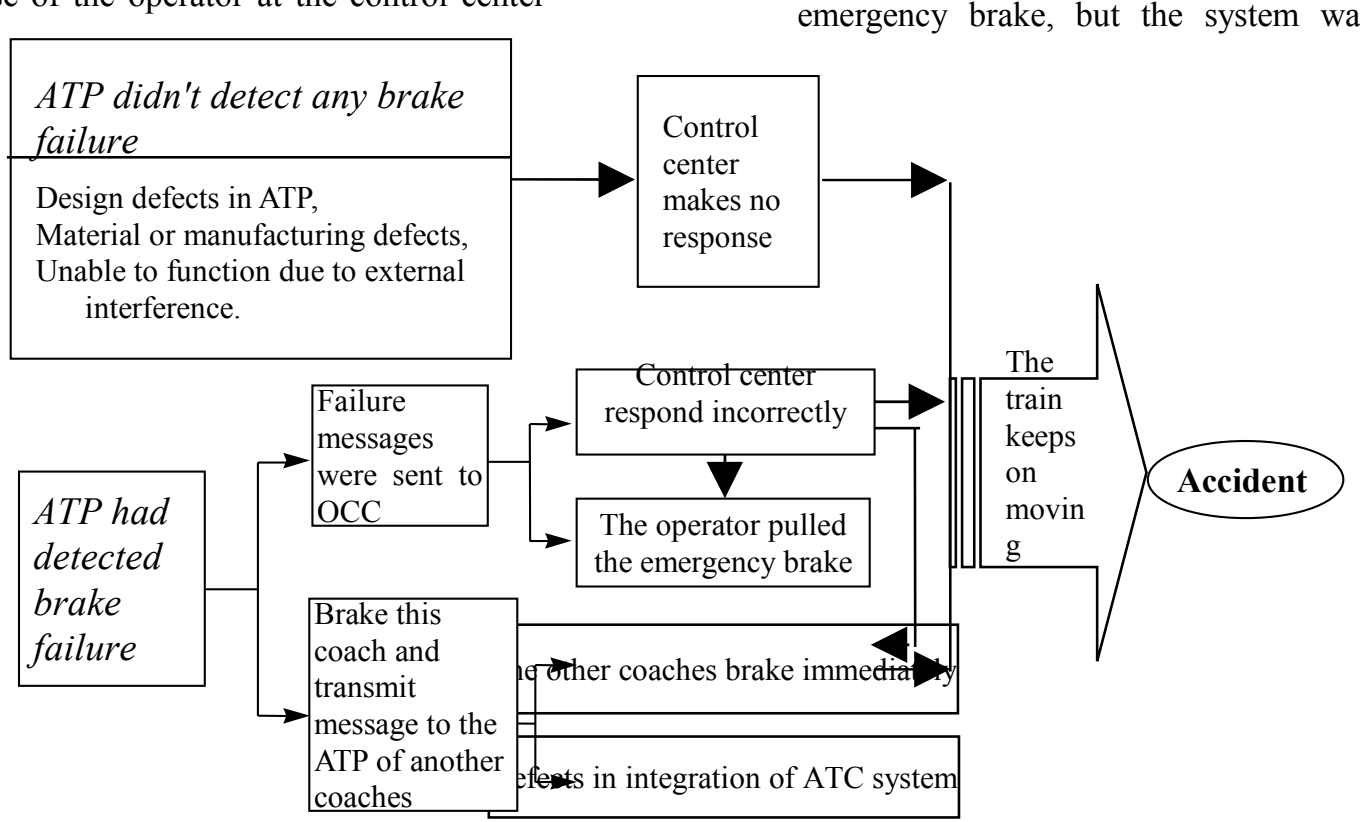

Fig. 7. Consequence diagram for hazards identification in the second stage analysis still fail to stop the exposure of hazards and the creation of hazardous medium.

(a) The operator at the control center made a wrong judgment and did not respond by pulling the brake after receiving the message from ATP. Two contradicting forces could be at work at this time. The operator is giving the command through ATO to allow the train to go forward, while the fail-safe features in ATP is pulling the train to come to a complete stop, hence the creation of a hazardous medium.

(b) The operator responded by pulling the emergency brake, but the system was 
integrating the systems between the two married-pair, i.e., one married pair was braking and the other was running forward, hence the creation of a hazardous medium.

The above analysis was verified in the report [10] that the cause of the September 24, 1993 fire accident was the defect in the design of connecting the married pair. After ATP detected TSR failure in one married pair, it immediately pulled the emergency brake for that system. However, the ATP located in the other married pair did not pick up the messages and carried on driving which led to the wearing of tires. After the train was dragged on for $2 \mathrm{~km}$, it eventually broke out in fire. This is the same as analyzed in the above: the defect integrating the systems between the two married-pair.

\section{DISCUSSION AND CONCLUSION}

The hazards identified in rail rapid transit are different with other transportation modes. Under the circumstance, the designing and management of the system are the major source of hazards and play critical roles for safety. The rail rapid transit safety features enable the automatic monitoring systems to eliminate the exposure of hazards immediately once the hazards are identified. While the hazards keep on exposing, the automatic monitoring system itself must be defective and could be regarded as another factors of hazards.

The model presented in this study analyses the process of hazard identification in two stages, which clearly indicates the factors of the hazard and the weaknesses in the response system. The two-stage analysis considering the inherent hazards in the first stage and the direct hazards in the second stage proposes a clear structure to go through the analyzing process and provides more information to the safety planner. When applying to accident causal analysis, the identification of hazards is not concluded with equipment causes, but include human and environment factors at the same time.

When the rail rapid transit accident has happened, the passive strategy derived from this hazard identification model is to recognize the defects in the analyzed system to formulate safety recommendations to improve transportation safety and stop the recurrence. The active or constructive plan of safety management is to control and eliminate the identified inherent hazards by farther improved managerial measures to prevent the accident from occurrence. Finally, what can be done after the identification of hazards? "It seems clear that the pursuit of safety must be pointed toward the identification of hazards, determination of their significance, evaluation of the available correctives, and selection of the optimal remedies. When this path is followed, it ends with the control of unwanted events at an irreducible minimum" (Grimaldi and Simonds, 1989). This is the practical view of accident prevention and the application of the model presented in this study can be the basis for further implementation for the transit authority.

\section{REFERENCES}

[1] FTA (Federal Transit Administration) National Transit Database. U.S. Department of Transportation, Washington DC. 1993, 1994, and 1995.

[2] J. V. Grimaldi and R. H. Simonds. Safety Management. 5th edition, Richard D. Irwin, Inc, Boston, MA. 1989.

[3] W. D. Keefer. "Circumstances surrounding accidents". In Industrial Safety, 2nd edition, eds R. P. Blake, pp. 45-59. Prentice-Hall, Inc, New Jersey. 1955.

[4] A. E. Florio, W. F. Alles and G. T. Stafford. Safety Education. 4th edition, McGraw-Hill, New York. 1979.

[5] H. W. Heinrich and E. R. Granniss. Industrial Accident Prevention: a Scientific Approach. 4th edition, McGraw-Hill, New York. 1959.

[6] NTSB (National Transportation Safety Board) Rail Rapid Transit Report. Washington, D.C. $1972 \sim 1981$

[7] San Francisco BART (Bay Area Rapid Transit District) Passenger Injuries Quarterly Report. Oakland Center, CA. $1985 \sim 1988$.

[8] L. C. Wang. "A Study of Rail Rapid Transit Hazards". Proceedings of the 8th Annual Conference of the Chinese Institute of Transportation, Taipei, Taiwan, R.O.C. pp. 8188. 1993.

[9] C. Y. Hwang. "VAL System Controlling Architecture." Rapid-Transit Technology Vol. 4, pp. 4-13. 1991.

[10] Department of Rapid Transit System, Taipei Rapid Transit Red Book -Mucha Line Evaluation Report. Taipei City Government, Taiwan, R.O.C. 1995 